Discrete Comput Geom 34:351-362 (2005)

DOI: $10.1007 / \mathrm{s} 00454-005-1161-7$

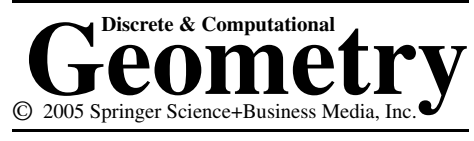

\title{
Stability of the Minkowski Measure of Asymmetry for Convex Bodies
}

\author{
Qi Guo \\ Department of Mathematics, Uppsala University, \\ Box 480, S-751 06 Uppsala, Sweden \\ guo@math.uu.se
}

Abstract. Given a convex body $C \subset R^{n}$ (i.e., a compact convex set with nonempty interior), for $x \in \operatorname{int}(C)$, the interior, and a hyperplane $H$ with $x \in H$, let $H_{1}, H_{2}$ be the two support hyperplanes of $C$ parallel to $H$. Let $r(H, x)$ be the ratio, not less than 1, in which $H$ divides the distance between $H_{1}, H_{2}$. Then the quantity

$$
A s(C):=\inf _{x \in \operatorname{int}(C)} \sup _{H \ni x} r(H, x)
$$

is called the Minkowski measure of asymmetry of $C$.

$A s(\cdot)$ can be viewed as a real-valued function defined on the family of all convex bodies in $R^{n}$. It has been known for a long time that $A s(\cdot)$ attains its minimum value 1 at all centrally symmetric convex bodies and maximum value $n$ at all simplexes.

In this paper we discuss the stability of the Minkowski measure of asymmetry for convex bodies. We give an estimate for the deviation of a convex body from a simplex if the corresponding Minkowski measure of asymmetry is close to its maximum value. More precisely, the following result is obtained:

Let $C \subset R^{n}$ be a convex body. If $A s(C) \geq n-\varepsilon$ for some $0 \leq \varepsilon<1 / 8(n+1)$, then there exists a simplex $S_{0}$ formed by $n+1$ support hyperplanes of $C$, such that

$$
(1+8(n+1) \varepsilon)^{-1} S_{0} \subset C \subset S_{0},
$$

where the homethety center is the (unique) Minkowski critical point of $C$. So

$$
d_{\mathrm{BM}}(C, S) \leq 1+8(n+1) \varepsilon
$$

holds for all simplexes $S$, where $d_{\mathrm{BM}}(\cdot, \cdot)$ denotes the Banach-Mazur distance.

\section{Introduction}

A measure of asymmetry (or symmetry) for convex bodies, according to Grünbaum's definition in his well-known paper [1], is a nonnegative real-valued function defined on 
the family of all convex bodies of a finite-dimensional (affine) space, which satisfies some additional conditions and therefore can be used to describe how close (or far) a convex body is to (or from) a centrally symmetric one. In [1] some known measures of symmetry were summarized and discussed, among which the Minkowski measure of asymmetry (see Section 2 in this paper or [5] for the definition) is probably the simplest and best known one. After [1] some new ones have also been studied.

Many important properties of such measures (especially the Minkowski measure) have been studied by different authors from different points of view (see [2]-[11]). Among these studies, Groemer [3] focuses on the stabilities of two measures of asymmetry that characterize cones as the most asymmetric convex bodies. More precisely, he established stability estimates for these (two) measures that provide information on the deviation of a convex body from a cone if the corresponding measure of symmetry is close to its maximum value. Naturally the same stability problems for other measures of asymmetry (or symmetry) should be studied too.

In this paper we study the stability of the Minkowski measure of asymmetry. We give an estimate for the deviation of a convex body from a simplex if the corresponding Minkowski measure of asymmetry is close to its maximum value.

The main result in this paper is the following stability theorem (the proof will be found in Section 4):

Theorem A. Let $C \subset R^{n}$ be a convex body. If its Minkowski measure of asymmetry As $(C) \geq n-\varepsilon$ for some $0 \leq \varepsilon \leq 1 / 8(n+1)$, then

$$
d_{\mathrm{BM}}(C, S) \leq 1+8(n+1) \varepsilon
$$

holds for all simplexes $S$, where $d_{\mathrm{BM}}(\cdot, \cdot)$ denotes the Banach-Mazur distance.

(See Section 2 for the definition of the Banach-Mazur distance). More precisely,

Theorem A*. Let $C \subset R^{n}$ be a convex body. If its Minkowski measure of asymmetry As $(C) \geq n-\varepsilon$ for some $0 \leq \varepsilon \leq 1 / 8(n+1)$, then there exists a simplex $S_{0}$ formed by $n+1$ support hyperplanes of $C$, such that

$$
(1+8(n+1) \varepsilon)^{-1} S_{0} \subset C \subset S_{0},
$$

where the homothety center is the (unique) Minkowski critical point of C.

Remark. The constant $8(n+1)$ here may not be the best, but there are examples showing that in general the term $\varepsilon$ cannot be replaced by $\varepsilon^{k}$ for any $k>1$.

\section{Preliminaries}

In this paper we work mainly on the $n$-dimensional Euclidean spaces $(n \geq 1)$ and work with affine maps (functions) instead of linear ones since it turns out that the affine setting works more suitably than the linear one. 
Denote by $R^{n}$ the standard Euclidean space, by $C, D$, etc., the convex sets in $R^{n}$ and by $\mathfrak{K}^{n}$ the family of all convex bodies (i.e., the compact convex sets with nonempty interiors) in $R^{n}$. The convex body of the form $\operatorname{conv}\left(x_{1}, \ldots, x_{n+1}\right)$ is called a simplex (in this case, $x_{1}, \ldots, x_{n+1}$ must be affinely independent), where conv denotes the convex hull. Notice that a simplex can be expressed as an intersection of suitable $n+1$ closed half-spaces. Denote by $T, S$, etc., the maps from $R^{n}$ to $R^{m}$.

Given $C \subset R^{n}$ and $T: R^{n} \rightarrow R^{m}$, denote by $T_{*}(C)$ the image set

$$
\left\{T x \in R^{m} \mid x \in C\right\} \text {. }
$$

A map $T: R^{n} \rightarrow R^{m}$ is called affine if $T\left(\sum_{i=1}^{k} \lambda_{i} x_{i}\right)=\sum_{i=1}^{m} \lambda_{i} T x_{i}$ for any $x_{i} \in R^{n}$ and $\lambda_{i} \in R(1 \leq i \leq k)$ with $\sum_{i=1}^{m} \lambda_{i}=1$. Especially, if $m=1, T$ is called an affine function. We usually denote functions by $f, g$, etc.

We denote

$$
\begin{aligned}
& \mathcal{A} f f\left(R^{n}, R^{m}\right):=\left\{T: R^{n} \rightarrow R^{m} \mid T \text { is affine }\right\}, \\
& \operatorname{aff}\left(R^{n}\right):=\left\{f \mid f \text { is an affine function on } R^{n}\right\} .
\end{aligned}
$$

aff $\left(R^{n}\right)$ is called the affine dual space of $R^{n}$, which is a linear space under the ordinary addition and scalar multiplication of functions and can be identified with $R^{n+1}$ in a natural way. We write $\mathcal{A} f f\left(R^{n}\right)$ in brief for $\mathcal{A} f f\left(R^{n}, R^{n}\right)$.

If we denote the linear dual space of aff $\left(R^{n}\right)$ by $\left(a f f\left(R^{n}\right)\right)^{\prime}$, then $R^{n}$ can be embedded as an affine subspace into $\left(\operatorname{aff}\left(R^{n}\right)\right)^{\prime}$. More precisely, under the correspondence $R^{n} \ni$ $x \leftrightarrow x^{\prime \prime} \in\left(\operatorname{aff}\left(R^{n}\right)\right)^{\prime}$ defined by $x^{\prime \prime}(f)=f(x)$ for $f \in \operatorname{aff}\left(R^{n}\right)$, we have

$$
R^{n}=\left\{z^{\prime \prime} \in\left(\operatorname{aff}\left(R^{n}\right)\right)^{\prime} \mid z^{\prime \prime}(\mathbf{1})=1\right\},
$$

where 1 denotes the constant affine function 1 on $R^{n}$.

For any $\lambda \in R, x \in R^{n}$, and $C \subset R^{n}$, we denote

$$
\lambda_{x} C:=\{x+\lambda(y-x) \mid y \in C\} .
$$

We recall the well-known Banach-Mazur distance defined on $\mathfrak{K}^{n}$.

Definition 1. Given $C, D \in \mathfrak{K}^{n}$, the Banach-Mazur distance $d_{\mathrm{BM}}(C, D)$ between $C, D$ is defined as

$$
d_{\mathrm{BM}}(C, D):=\inf \left\{\lambda>0 \mid T_{*}(C) \subset D \subset \lambda_{x}\left(T_{*}(C)\right)\right\},
$$

where the infimum is taken over all $x \in R^{n}$ and all nonsingular $T \in \mathcal{A} f f\left(R^{n}\right)$.

Remark 1. It is easy to see that $d_{\mathrm{BM}}(C, D)=1$ iff $C$ and $D$ are affinely equivalent, i.e., there exists a nonsingular $T \in \mathcal{A} f f\left(R^{n}\right)$ such that $D=T_{*}(C)$. Thus $d_{\mathrm{BM}}$ (or more precisely $\log d_{\mathrm{BM}}$ ) is actually a metric on $\mathfrak{K}^{n} / \sim$, where " $\sim$ " denotes the affine equivalence relation.

In this paper the main objects we study are the so-called (affinely invariant) measures of asymmetry for convex bodies. So we recall here some measures of asymmetry (for a general definition of measures of asymmetry, see [1] or [5]). 
Definition 2 (see [1]). Given $C \in \mathfrak{K}^{n}$, for $x \in \operatorname{int}(C)$, the interior, and a hyperplane $H$ with $x \in H$, let $H_{1}, H_{2}$ be the two support hyperplanes of $C$ parallel to $H$. Let $r(H, x)$ be the ratio, not less than 1 , in which $H$ divides the distance between $H_{1}, H_{2}$. Now if we denote

$$
r(x)=r(C, x):=\sup _{H \ni x} r(H, x),
$$

then the Minkowski measure of asymmetry of $C$ is defined as

$$
A s(C):=\inf _{x \in \operatorname{int}(C)} r(x) .
$$

Any point $c \in \operatorname{int}(C)$ satisfying $r(c)=A s(C)$ is called a (Minkowski) critical point of $C$.

Remark 2. (1) It is easy to see that $A s(\cdot)$ is affinely invariant, i.e., $A s\left(T_{*}(C)\right)=A s(C)$ for any $C \in \mathfrak{K}^{n}$ and any nonsingular $T \in \mathcal{A} f f\left(R^{n}\right)$.

(2) It is easy to check that the function $r(x)$ defined on $\operatorname{int}(C)$ is convex (see Remark 4) and $\lim _{x \rightarrow \partial C} r(x)=\infty$, where $\partial C$ denotes the boundary of $C$, so $\inf _{x \in \operatorname{int}(C)} r(x)$ is attained, i.e., for each $C$ there exists at least one critical point.

(3) There are several other equivalent definitions. For instance, for any $x \in \operatorname{int}(C)$ and chord $l$ of $C$ passing through $x$, let $r^{\prime}(l, x)$ be the ratio, not less than 1 , in which $x$ divides the length of $l$. Then it is known that $r(x)=\sup _{l \ni x} r^{\prime}(l, x)$, and so

$$
A s(C)=\inf _{x \in \operatorname{int}(C)} \sup _{l \ni x} r^{\prime}(l, x) .
$$

For other equivalent definitions see [6] or [9].

(4) It has been known for a long time that $1 \leq A s(C) \leq n\left(\forall C \in \mathfrak{K}^{n}\right)$ and $A s(C)=1$ iff $C$ is centrally symmetric; $A s(C)=n$ iff $C$ is a simplex (see [1] or [2]).

The following measure of symmetry, introduced for the first time in [9], is equivalent in some sense to the Minkowski one and is more practical and useful. To introduce this measure of symmetry, we first give the concept of an affine dual set of a given $C \in \mathfrak{K}^{n}$ (see [9]).

Definition 3 (see [9]). Given $C \in \mathfrak{K}^{n}$, we define the affine dual set $C_{[0,1]}^{A}$ of $C$ by

$$
C_{[0,1]}^{A}:=\left\{f \in \operatorname{aff}\left(R^{n}\right) \mid f_{*}(C) \subset[0,1]\right\} .
$$

We also denote

$$
C_{[0,1]}^{a}:=\left\{f \in \operatorname{aff}\left(R^{n}\right) \mid f_{*}(C)=[0,1]\right\},
$$

which is a subset of $\partial C_{[0,1]}^{A}$.

Remark 3. $C_{[0,1]}^{a}$ consists of the main part of $\partial\left(C_{[0,1]}^{A}\right)$ in the sense that $C_{[0,1]}^{A}=$ $\operatorname{conv}\left(0,1, C_{[0,1]}^{a}\right)$, where 0,1 denote the constant affine functions $\mathbf{0}, \mathbf{1}$ on $R^{n}$, respectively. 
Now we have the following

Definition 4 (see [9] or [10]). Given $C \in \mathfrak{K}^{n}$. For each $x \in \operatorname{int}(C)$, denote

$$
v(x)=v(C, x)=\inf _{f \in C_{[0,1]}^{a}} f(x),
$$

and we define the measure $a s(C)$ of symmetry of $C$ by

$$
\operatorname{as}(C):=\sup _{x \in \operatorname{int}(C)} v(C, x) .
$$

Any point $x$ satisfying $v(C, x)=a s(C)$ is called an $a s$-critical point of $C$.

Remark 4. (1) For each $C \in \mathfrak{K}^{n}$, the function $v(C, x)$ defined on $C$ is concave since it is the infimum of a family of affine functions, and $v(C, x)_{\mid \partial C}=0$. So $a s(C)$ is attained, i.e., there exists at least one $a s$-critical point for each $C$.

(2) It is not hard to see that for any $C \in \mathfrak{K}^{n}$ and $x \in \operatorname{int}(C)$, we have

$$
r(x)=\frac{1}{v(x)}-1 \quad \text { or } \quad v(x)=\frac{1}{r(x)+1},
$$

and consequently

$$
A s(C)=\frac{1}{a s(C)}-1 \quad \text { or } \quad a s(C)=\frac{1}{A s(C)+1}
$$

from which and (4) in Remark 2 it follows that $C$ is symmetric iff $a s(C)=\frac{1}{2}, C$ is a simplex iff $\operatorname{as}(C)=1 /(n+1)$, and $c \in \operatorname{int}(C)$ is a Minkowski critical point iff $c$ is an as-critical point.

We also point out that, with the help of the relation between $r(x)$ and $v(x)$ above, the convexity of the function $r(x)$ in Definition 2 can be easily derived from the concavity of the function $v(x)$.

We use $\mathcal{C}_{C}$ to denote the set of all as critical (or Minkowski) points of $C$. By the above, $\mathcal{C}_{C}$ is a nonempty convex set.

\section{Some Properties of the Critical Affine Functionals}

Given a convex body $C \in \mathfrak{K}^{n}$ and $c \in \mathcal{C}_{C}$, an affine function $f \in C_{[0,1]}^{a}$ is called a critical function (with respect to $c$ ) of $C$ if $f(c)=a s(C)$.

We point out that if $f$ is a critical function, then the ratio, not less than 1 , in which $H:=\{f=a s(C)\}$ divides the distance between $H_{0}:=\{f=0\}$ and $H_{1}:=\{f=1\}$, is exactly the Minkowski measure $A s(C)$. Therefore the hyperplane $\{f=0\}$ is called a critical support hyperplane (with respect to $c$ ) of $C$ if $f$ is a critical function (with respect to $c$ ) of $C$.

Denote, for a fixed $c \in \mathcal{C}_{C}$, by $\mathbb{E}_{C}(c)$ the family of all critical functions with respect to $c$, i.e.,

$$
\mathbb{E}_{C}(c)=\left\{f \in C_{[0,1]}^{a} \mid f(c)=\operatorname{as}(C)\right\}
$$


In this section we mainly discuss the properties of $\mathbb{E}_{C}(c)$. We show that $\mathbb{E}_{C}(c)$ contains "enough" elements to represent some constant functions, which in turn implies that, under some conditions, there exist "enough" critical support hyperplanes to form a simplex which is "close to" $C$.

Lemma 1. $C_{[0,1]}^{a}$ is bounded and closed, and so is its convex hull conv $\left(C_{[0,1]}^{a}\right)$.

Proof. The boundedness is obvious. So we need only show the closedness.

Suppose $f_{0} \in \operatorname{aff}\left(R^{n}\right), f_{k} \in C_{[0,1]}^{a}(k=1,2, \ldots)$ satisfy

$$
\lim _{k \rightarrow \infty} f_{k}(x)=f_{0}(x) \quad \text { for all } \quad x \in R^{n} .
$$

First, it is clear that $f_{0 *}(C) \subset[0,1]$. To show the converse inclusion, we choose, for each $k, x_{k}, y_{k} \in C$ such that $f_{k}\left(x_{k}\right)=0, f_{k}\left(y_{k}\right)=1$. Then by the compactness of $C$, we may find $x_{0}, y_{0} \in C$ such that (by passing to subsequences)

$$
x_{k} \rightarrow x_{0}, \quad y_{k} \rightarrow y_{0}, \quad k \rightarrow \infty .
$$

Then by the equicontinuity of $f_{k}$ on $C$, it follows that we must have $f_{0}\left(x_{0}\right)=\lim _{k \rightarrow \infty}$ $f_{k}\left(x_{0}\right)=\lim _{k \rightarrow \infty} f_{k}\left(x_{k}\right)=0$ and similarly $f_{0}\left(y_{0}\right)=1$. Therefore $f_{0 *}(C) \supset[0,1]$. Thus $f_{0 *}(C)=[0,1]$, i.e., $f_{0} \in C_{[0,1]}^{a}$.

If we regard each number in $R$ as a constant affine function on $R^{n}$, then we have the following:

Lemma 2. For any $C \in \mathfrak{K}^{n}$, we have

$$
\operatorname{conv}\left(C_{[0,1]}^{a}\right) \cap R=[\operatorname{as}(C), 1-\operatorname{as}(C)] .
$$

Proof. We first observe that $\mu \in \operatorname{conv}\left(C_{[0,1]}^{a}\right)$ iff $1-\mu \in \operatorname{conv}\left(C_{[0,1]}^{a}\right)$. In fact, if $\mu \in$ $\operatorname{conv}\left(C_{[0,1]}^{a}\right)$, i.e., $\mu=\sum_{i=1}^{N} \alpha_{i} f_{i}$ for some $f_{i} \in C_{[0,1]}^{a}$ and $\alpha_{i}>0$ with $\sum_{i=1}^{N} \alpha_{i}=1$, then $1-\mu=\sum_{i=1}^{N} \alpha_{i}\left(1-f_{i}\right) \in \operatorname{conv}\left(C_{[0,1]}^{a}\right)$ since if $f \in C_{[0,1]}^{a}$, then $1-f \in C_{[0,1]}^{a}$. Then we just need to change the roles of $\mu$ and $1-\mu$.

Now suppose $\mu \in \operatorname{conv}\left(C_{[0,1]}^{a}\right)$, i.e., $\mu=\sum_{i=1}^{N} \alpha_{i} f_{i}$ for some $f_{i} \in C_{[0,1]}^{a}$ and $\alpha_{i}>$ 0 with $\sum_{i=1}^{N} \alpha_{i}=1$, then by choosing $c \in \mathcal{C}_{C}$, we get that $\mu=\sum_{i=1}^{N} \alpha_{i} f_{i}(c) \geq$ $\sum_{i=1}^{N} \alpha_{i}$ as $(C)=\operatorname{as}(C)$. Thus by the above observation, we proved in fact that

$$
\operatorname{conv}\left(C_{[0,1]}^{a}\right) \cap R \subset[\operatorname{as}(C), 1-\operatorname{as}(C)] .
$$

To prove the converse inclusion, by the same observation above, we now need only show that $\operatorname{as}(C) \in \operatorname{conv}\left(C_{[0,1]}^{a}\right)$. To see this, we first make another observation that the constant function $\frac{1}{2} \in \operatorname{conv}\left(C_{[0,1]}^{a}\right)$ since $\frac{1}{2}=\frac{1}{2} f+\frac{1}{2}(1-f)$ for any $f \in C_{[0,1]}^{a}$.

Now suppose $\operatorname{as}(C) \notin \operatorname{conv}\left(C_{[0,1]}^{a}\right)$, then by the Hahn-Banach theorem, there is $x^{\prime \prime} \in$ $\left(\text { aff }\left(R^{n}\right)\right)^{\prime}$ such that

$$
x^{\prime \prime}(\operatorname{as}(C))<\inf _{f \in \operatorname{conv}\left(C_{[0,1]}^{a}\right)} x^{\prime \prime}(f) .
$$


Notice that $x^{\prime \prime}(\mathbf{1}) \neq 0$, otherwise we will have $x^{\prime \prime}(\lambda)=\lambda x^{\prime \prime}(\mathbf{1})=0$ for all $\lambda \in R$ and especially $x^{\prime \prime}(\operatorname{as}(C))=x^{\prime \prime}\left(\frac{1}{2}\right)=0$ which contradicts (3.1) since $\frac{1}{2} \in \operatorname{conv}\left(C_{[0,1]}^{a}\right)$.

We denote $x^{*}=x^{\prime \prime} / x^{\prime \prime}(\mathbf{1})$. Thus we have that $x^{*}(\mathbf{1})=1$, which ensures by (2.1) that $x^{*} \in R^{n}$, and that either

$$
\operatorname{as}(C)=x^{*}(\operatorname{as}(C))<\inf _{f \in C_{[0,1]}^{a}} x^{*}(f)=\inf _{f \in C_{[0,1]}^{a}} f\left(x^{*}\right)
$$

or

$$
\operatorname{as}(C)=x^{*}(\operatorname{as}(C))>\sup _{f \in C_{[0,1]}^{a}} x^{*}(f)=\sup _{f \in C_{[0,1]}^{a}} f\left(x^{*}\right) .
$$

Since $x^{*} \in R^{n}$, then either $x^{*} \in C$ or $x^{*} \notin C$. If $x^{*} \notin C$, then by the Hahn-Banach theorem, in case (3.2), we may choose $f_{0} \in C_{[0,1]}^{a}$ such that $f_{0}\left(x^{*}\right)<0$ which leads to the contradiction that $a s(C)<0$, and, in case (3.3), we may choose $f_{1} \in C_{[0,1]}^{a}$ such that $f_{1}\left(x^{*}\right)>1$ which leads to the contradiction that $a s(C)>1$. So we must have $x^{*} \in C$.

However, if $x^{*} \in C$, then (3.2) implies that

$$
\operatorname{as}(C)<v\left(C, x^{*}\right),
$$

which is a contradiction to the definition of $a s(C)$. Equation (3.3) implies the contradiction that $\operatorname{as}(C)>\frac{1}{2}$ since we can always choose $f_{2} \in C_{[0,1]}^{a}$ such that $f_{2}\left(x^{*}\right) \geq \frac{1}{2}$. Therefore we must have that $\operatorname{as}(C) \in \operatorname{conv}\left(C_{[0,1]}^{a}\right)$. This completes the proof.

Remark 5. Since $1 /(n+1) \leq a s(C) \leq \frac{1}{2}$ for $C \in \mathfrak{K}^{n}$, by Lemma 2 , we get that

$$
\left\{\frac{1}{2}\right\} \subset \operatorname{conv}\left(C_{[0,1]}^{a}\right) \cap R \subset\left|\frac{1}{n+1}, \frac{n}{n+1}\right| .
$$

Lemma 3. If as $(C)=\sum_{i=1}^{N} \alpha_{i} f_{i}$ for $f_{i} \in C_{[0,1]}^{a}$ and $\alpha_{i}>0$ with $\sum_{i=1}^{N} \alpha_{i}=1$, then for any $c \in \mathcal{C}_{C}, f_{i} \in \mathbb{E}_{C}(c)$ for all $i$.

Proof. For each $c \in \mathcal{C}_{C}$, we have

$$
\operatorname{as}(C)=\sum_{i=1}^{N} \alpha_{i} f_{i}(c) \geq \sum_{i=1}^{N} \alpha_{i} \operatorname{as}(C)=\operatorname{as}(C)
$$

since $f_{i}(c) \geq a s(C)$ for all $i$, which in turn implies that $f_{i}(c)=a s(C)$ for all $i$.

By Lemmas 2 and 3, we immediately get

Corollary 1. For any $C \in \mathfrak{K}^{n}$ and $c \in \mathcal{C}_{C}$, we have

$$
\operatorname{as}(C) \in \operatorname{conv}\left(\mathbb{E}_{C}(c)\right) .
$$


Furthermore, we have the following:

Lemma 4. For any $c \in \mathcal{C}_{C}$, there exist $\left\{f_{i}\right\}_{i=1}^{n+1} \subset \mathbb{E}_{C}(c)$ and $\alpha_{1} \geq 0, \ldots, \alpha_{n+1} \geq 0$ with $\sum_{i=1}^{n+1} \alpha_{i}=1$ such that

$$
\operatorname{as}(C)=\sum_{i=1}^{n+1} \alpha_{i} f_{i}
$$

Proof. By Corollary 1, as $(C) \in \operatorname{conv}\left(\mathbb{E}_{C}(c)\right)$. However, $\mathbb{E}_{C}(c)$ is a subset of the $n$ dimensional affine space $\left\{f \in \operatorname{aff}\left(R^{n}\right) \mid f(c)=a s(C)\right\}$, thus by the Carathéodory theorem there exist $\left\{f_{i}\right\}_{i=1}^{n+1} \subset \mathbb{E}_{C}(c)$ and $\alpha_{i} \geq 0$ with $\sum_{i=1}^{n+1} \alpha_{i}=1$ such that

$$
\operatorname{as}(C)=\sum_{i=1}^{n+1} \alpha_{i} f_{i}
$$

Lemma 5. If as $(C)=\sum_{i=1}^{N} \alpha_{i} f_{i}$ for $f_{i} \in C_{[0,1]}^{a}$ and $\alpha_{i}>0$ with $\sum_{i=1}^{N} \alpha_{i}=1$, then $\alpha_{i} \leq$ as $(C)$ for all $i$. Therefore $\alpha_{i} \geq 1-(N-1)$ as $(C)$ for all $i$.

Proof. For each $i(1 \leq i \leq N)$, choose $x_{i} \in \partial C$ such that $f_{i}\left(x_{i}\right)=1$, then (notice that $f_{j}\left(x_{i}\right) \geq 0$ for all $\left.i, j\right)$ we have for each $i$,

$$
\operatorname{as}(C)=\sum_{j=1}^{N} \alpha_{j} f_{j}\left(x_{i}\right)=\alpha_{i}+\sum_{j \neq i} \alpha_{j} f_{j}\left(x_{i}\right) \geq \alpha_{i}
$$

and for each $i$,

$$
\alpha_{i}=1-\sum_{j \neq i}^{N} \alpha_{j} \geq 1-(N-1) \operatorname{as}(C)
$$

Lemma 6. If as $(C)<1 / n$ and if as $(C)=\sum_{i=1}^{n+1} \alpha_{i} f_{i}$ for some $f_{i} \in \mathbb{E}_{C}(c)$ (where $c$ is the unique critical point of $C$ ) and $\alpha_{i} \geq 0$ with $\sum_{i=1}^{n+1} \alpha_{i}=1$, then the set $\Delta:=$ $\bigcap_{i=1}^{n+1}\left\{f_{i} \geq 0\right\}$ is bounded, i.e., $\Delta$ is a simplex.

Proof. Before we prove the lemma, we point out that, under the assumption that as $(C)<1 / n$, the uniqueness of the critical point is ensured by a theorem in [2] which states that $\operatorname{dim} \mathcal{C}_{C}+a s(C)^{-1} \leq n+1$, where dim denotes the dimension.

To complete the proof, we first notice that, by Lemma $5, \alpha_{i} \geq 1-n \operatorname{as}(C)>$ $1-n(1 / n)=0$ for all $i$.

Thus, suppose $\Delta$ is not bounded, then there exists a ray $l \subset \Delta$. Now if there exists some $1 \leq i_{0} \leq n+1$, say $i_{0}=1$, such that $f_{1}$ is not a constant on $l$, then we will have $\sup _{x \in l} f_{1}(x)=+\infty$ since $f_{1}$ is an affine function and $f_{1}(x) \geq 0$ on $l \subset \Delta$, which, together with the fact that for any $x \in l$, as $(C)=\alpha_{1} f_{1}(x)+\sum_{i=2}^{n+1} \alpha_{i} f_{i}(x) \geq$ $\alpha_{1} f_{1}(x)$ since $f_{i}(x) \geq 0$ for all $i$ and $x \in l$, leads to the contradiction that $\operatorname{as}(C) \geq$ $\sup _{x \in l} \alpha_{1} f_{1}(x)=+\infty$. So all $f_{i}$ are constants on $l$.

Now we consider the $(n-1)$-dimensional space $R^{n} /[l] \cong R^{n-1}$ where $[l]$ denotes the 
one-dimensional affine space containing $l$. Let $P: R^{n} \rightarrow R^{n-1}$ be the corresponding projection, and denote $\widetilde{C}=P(C)=\{\tilde{x} \mid \tilde{x}=P(x), x \in C\}$. It is clear that $\widetilde{C}$ is a convex body in $R^{n-1}$. Now if we define $\tilde{f}_{i} \in$ aff $\left(R^{n-1}\right)(1 \leq i \leq n+1)$ by $\tilde{f}_{i}(\tilde{x}):=f_{i}(x)$ for $\tilde{x} \in R^{n-1}$, then it is easy to see that $\tilde{f}_{i} \in \widetilde{C}_{[0,1]}^{a}(1 \leq i \leq n+1)$ and that $\operatorname{as}(C)=\sum_{i=1}^{n+1} \alpha_{i} \tilde{f}_{i}$, i.e., $\operatorname{as}(C) \in \operatorname{conv}\left(\widetilde{C}_{[0,1]}^{a}\right)$, which, together with the fact that $a s(C)<1 / n \leq 1 / 2$, implies that $\frac{1}{n} \in \operatorname{conv}\left(\widetilde{C}_{[0,1]}^{a}\right)$, a contradiction to Lemma 2 (see Remark 5). This finishes the proof.

Now, by Lemmas 4 and 6, we have the following:

Corollary 2. Let $C \in \mathfrak{K}^{n}$. If as $(C)<1 / n$, then there exist $f_{i} \in \mathbb{E}_{C}(c)(1 \leq i \leq n+1)$, where (the unique) $c \in \mathcal{C}_{C}$, such that $\bigcap_{i=1}^{n+1}\left\{f_{i} \geq 0\right\}$ is bounded, i.e., $\bigcap_{i=1}^{n+1}\left\{f_{i} \geq 0\right\}$ is a simplex.

\section{Proofs of the Main Theorems}

We first show a simple property of the critical functions of a simplex.

Let $S$ be a simplex with facets $F_{i}(1 \leq i \leq n+1)$. We denote by $f_{F_{i}} \in S_{(0,1]}^{a}$ the affine function vanishing on $F_{i}$. Then we have

Lemma 7. If $S$ is a simplex with facets $F_{i}(1 \leq i \leq n+1)$, then

$$
\sum_{i=1}^{n+1} f_{F_{i}}=1
$$

Proof. If we denote by $x_{i}$ the unique point in $\left\{f_{F_{i}}=1\right\} \cap S(1 \leq i \leq n+1)$, i.e., $x_{i}$ $(1 \leq i \leq n+1)$ are all the vertices of $S$, then we have that $f_{F_{i}}\left(x_{i}\right)=1$ and $f_{F_{i}}\left(x_{j}\right)=0$ for $j \neq i$. Furthermore, it is known that for each $x \in R^{n}$, there exist $\beta_{i} \in R(1 \leq i \leq n+1)$ such that $\sum_{i=1}^{n+1} \beta_{i}=1$ and $x=\sum_{i=1}^{n+1} \beta_{i} x_{i}$. Therefore

$$
\begin{aligned}
\sum_{i=1}^{n+1} f_{F_{i}}(x) & =\sum_{j=1}^{n+1} f_{F_{j}}\left(\sum_{i=1}^{n+1} \beta_{i} x_{i}\right)=\sum_{i, j=1}^{n+1} \beta_{i} f_{F_{j}}\left(x_{i}\right) \\
& =\sum_{i=1}^{n+1} \beta_{i} f_{F_{i}}\left(x_{i}\right)=\sum_{i=1}^{n+1} \beta_{i}=1 .
\end{aligned}
$$

Notice that the functions $f_{F_{i}}$ in Lemma 7 are actually critical functions of the simplex. So we give the following stability version of Lemma 7 for general convex bodies.

Lemma 8. Let $C \in \mathfrak{K}^{n}, c \in \mathcal{C}_{C}$, if as $(C) \leq(1 /(n+1))(1+\varepsilon)$ for some $0 \leq \varepsilon<$ $1 / 2(n+1)$, then for any $n+1$ affine functions $f_{1}, \ldots, f_{n+1} \in \mathbb{E}_{C}(c)$ satisfying that $\bigcap_{i=1}^{n+1}\left\{f_{i} \geq 0\right\}$ is bounded (i.e., a simplex), we have

$$
1 \leq \sum_{i=1}^{n+1} f_{i}(x) \leq 1+2(n+1) \varepsilon \quad \text { for all } \quad x \in \bigcap_{i=1}^{n+1}\left\{f_{i} \geq 0\right\} .
$$


Proof. Denote by $S$ the simplex $\bigcap_{i=1}^{n+1}\left\{f_{i} \geq 0\right\}(\supset C)$, and denote by $g_{i}(1 \leq i \leq n+1)$, where $g_{i}$ satisfies $\left\{g_{i}=0\right\}=\left\{f_{i}=0\right\}$, the critical functional of $S$. Then by Lemma 7 and the fact that $g_{i}(x) \leq f_{i}(x)$ for all $x \in S$, it follows that

$$
1=\sum_{i=1}^{n+1} g_{i}(c) \leq \sum_{i=1}^{n+1} f_{i}(c)=(n+1) \text { as }(C) \leq 1+\varepsilon .
$$

So

$$
0 \leq \sum_{i=1}^{n+1}\left(f_{i}(c)-g_{i}(c)\right) \leq \varepsilon .
$$

Thus, for any $1 \leq i \leq n+1$,

$$
g_{i}(c) \geq f_{i}(c)-\varepsilon .
$$

Therefore, for all $x \in S$ (notice that $\left\{g_{i}=0\right\}=\left\{f_{i}=0\right\}$ ), we have

$$
\begin{aligned}
g_{i}(x) & =\frac{g_{i}(c)}{f_{i}(c)} f_{i}(x) \geq \frac{f_{i}(c)-\varepsilon}{f_{i}(c)} f_{i}(x) \\
& =\left(1-\frac{1}{f_{i}(c)} \varepsilon\right) f_{i}(x) \geq(1-(n+1) \varepsilon) f_{i}(x)
\end{aligned}
$$

(here we use the fact that $f_{i}(c)=a s(C) \geq 1 /(n+1)$ ) followed by

$$
f_{i}(x) \leq(1-(n+1) \varepsilon)^{-1} g_{i}(x) \leq(1+2(n+1) \varepsilon) g_{i}(x),
$$

where we use the fact that $(1-t)^{-1} \leq 1+2 t$ for $0 \leq t \leq \frac{1}{2}$. Now it follows that, for all $x \in S$,

$$
\begin{aligned}
1 & =\sum_{i=1}^{n+1} g_{i}(x) \leq \sum_{i=1}^{n+1} f_{i}(x) \\
& \leq(1+2(n+1) \varepsilon) \sum_{i=1}^{n+1} g_{i}(x)=1+2(n+1) \varepsilon .
\end{aligned}
$$

The next theorem, stated as a stability theorem for the as-measure of asymmetry, is equivalent to Theorem $\mathrm{A}^{*}$.

Theorem 1. Given $C \in \mathfrak{K}^{n}$. If as $(C) \leq(1 /(n+1))(1+\varepsilon)$ for some $0 \leq \varepsilon<$ $1 / 4(n+1)^{2}$, then there exists a simplex $S_{0}$ formed by the critical support hyperplanes such that

$$
\left(1+4(n+1)^{2} \frac{\varepsilon}{n}\right)_{c}^{-1} S_{0} \subset C \subset S_{0} .
$$

Proof. We will show actually that for all simplexes $S$ of the form $\bigcap_{i=1}^{n+1}\left\{f_{i} \geq 0\right\}$, where $f_{i} \in \mathbb{E}_{C}(c)(1 \leq i \leq n+1)$, we have

$$
\left(1+4(n+1)^{2} \frac{\varepsilon}{n}\right)_{c}^{-1} S \subset C \subset S,
$$


which together with Corollary 2 (notice that the assumption on $\varepsilon$ ensures that $(1 /(n+$ 1)) $(1+\varepsilon)<1 / n)$ will finish the proof.

Suppose a simplex $S_{0}=\bigcap_{i=1}^{n+1}\left\{f_{i} \geq 0\right\}$ for some $f_{i} \in \mathbb{E}_{C}(c)(1 \leq i \leq n+1)$, then by Lemma 8 , for all $x \in C$,

$$
1 \leq \sum_{i=1}^{n+1} f_{i}(x) \leq 1+2(n+1) \varepsilon .
$$

Therefore, for any fixed $1 \leq j \leq n+1$, if we choose $y_{j} \in C \cap\left\{f_{j}=1\right\}$, then we have

$$
\sum_{i \neq j} f_{i}\left(y_{j}\right)+1 \leq 1+2(n+1) \varepsilon \quad \text { or } \quad \sum_{i \neq j} f_{i}\left(y_{j}\right) \leq 2(n+1) \varepsilon
$$

followed by

$$
f_{i}\left(y_{j}\right) \leq 2(n+1) \varepsilon, \quad i \neq j .
$$

Now set $S_{1}=\bigcap_{i=1}^{n+1}\left\{f_{i} \geq 2(n+1) \varepsilon\right\}$. Then clearly $S_{1}$ is bounded since $S_{1} \subset S_{0}$, and $\operatorname{int}\left(S_{1}\right) / \varphi$ since $c \in \operatorname{int}\left(S_{1}\right)$ derived from the fact that $f_{i}(c) \geq 1 /(n+1)>2(n+1) \varepsilon$ for all $i$. So $S_{1}$ is a simplex. It is also clear that the facets $\left\{f_{i}=2(n+1) \varepsilon\right\}$ of $S_{1}$ are parallel to the facets $\left\{f_{i}=0\right\}$ of $S_{0}$.

Furthermore, since for any $1 \leq i \leq n+1$,

$$
\frac{f_{i}(c)}{f_{i}(c)-2(n+1) \varepsilon}=1+\frac{2(n+1)}{f_{i}(c)-2(n+1) \varepsilon} \varepsilon<1+4(n+1)^{2} \varepsilon
$$

(where we used the fact that $f_{i}(c)-2(n+1) \varepsilon>1 /(n+1)-2(n+1) / 4(n+1)^{2}=$ $1 / 2(n+1))$, we have

$$
\lambda_{c}^{-1} S_{0} \subset S_{1}
$$

where $\lambda=1+4(n+1)^{2} \varepsilon$.

Notice further that (since $f_{i}\left(y_{j}\right) \leq 2(n+1) \varepsilon$ for all $1 \leq j \leq n+1, i \neq j$ )

$$
S_{1} \subset \operatorname{conv}\left\{y_{j} \mid 1 \leq j \leq n+1\right\} \subset C,
$$

we finally get

$$
\lambda_{c}^{-1} S_{0} \subset S_{1} \subset C \subset S_{0}
$$

This completes the proof.

Now we can finish the proof for Theorem $A^{*}$ :

Proof of Theorem $A^{*}$. By Remark 4, it is easy to see that if $A s(C) \geq n-\varepsilon$, then as $(C) \leq(1 /(n+1))(1+\varepsilon / n)$, and $\varepsilon / n<1 / 4(n+1)^{2}$ when $\varepsilon<1 / 8(n+1)$. So by Theorem 1 and the fact that $1+4(n+1)^{2}(\varepsilon / n) \leq 1+8(1+n) \varepsilon$ when $\varepsilon<1 / 8(n+1)$, there is a simplex $S_{0}$ formed by the critical support hyperplanes of $C$ such that

$$
(1+8(1+n) \varepsilon)_{c}^{-1} S_{0} \subset\left(1+4(n+1)^{2} \frac{\varepsilon}{n}\right)_{c}^{-1} S_{0} \subset C \subset S_{0} .
$$


The following estimate immediately comes from Theorem A.

Corollary 3. Let $C, D \in \mathfrak{K}^{n}$. If $A s(C) \geq n-1 / 8(n+1)$, As $(D) \geq n-1 / 8(n+1)$, then

$$
d_{M}(C, D) \leq 1+c_{n} \max \{n-A s(C), n-A s(D)\},
$$

where $c_{n}=24(n+1)$.

\section{Acknowledgments}

The author expresses his heartfelt thanks to his supervisor Sten Kaijser for his invaluable and motivative suggestions and comments, especially for simplifying the many proofs in Section 3, during the writing of this paper. He also thanks the referees for their suggestions on the presentation and for pointing out some language mistakes.

\section{References}

1. B. Grünbaum, Measure of symmetry of convex sets, in Convexity, Proceedings of Symposia in Pure Mathematics 7, American Mathematical Society, Providence, 1963, pp. 233-270.

2. V.L. Klee, Jr, The critical set of a convex set, Amer. J. Math. 75 (1953), 178-188.

3. H. Groemer, Stability theorems for two measures of symmetry, Discrete Comput. Geom. 24 (2000), 301-311.

4. H. Groemer, L.J. Wallen, A measure of asymmetry for domains of constant width, Beiträge Algebra Geom. 42(2) (2001) 517-521.

5. Q. Guo, On $p$-measure of asymmetry for convex bodies, Manuscript.

6. Q. Guo, S. Kaijser, On asymmetry of some convex bodies, Discrete Comput. Geom. 27 (2002), 239-247.

7. Q. Guo, S. Kaijser, Approximations of convex bodies by convex bodies, Northeast. Math. J. 19(4) (2003), 323-332.

8. S.S. Kutateladze, Symmetry measures, Math. Notes 19(3-4) (1976), 372-375.

9. S. Kaijser, Q. Guo, An estimate of the affine distance between convex bodies, U.U.D.M. Report 1992:16 (preprint).

10. Q. Guo, S. Kaijser, On the distance between convex bodies, Northeast. Math. J. 15(3) (1999), 323-331.

11. E. Ekström, The Critical Set of a Convex Body, U.U.D.M. Project Report 2000:P7.

Received June 2, 2004, and in revised form September 16, 2004. Online publication March 14, 2005. 